\section{GSR Journal}

Georgetown Scientific Research Journal
Volume 1 | Edition 2

\section{July 31, 2021}



GEORGETOWN SCIENTIFIC RESEARCH JOURNAL
Volume One

Edition Two

Spring 2021

\section{Cancer Models to Defeat Therapy Resistance in Pancreatic Ductal Adenocarcinoma}

Britney He 


\title{
Cancer Models to Defeat Therapy Resistance in Pancreatic Ductal Adenocarcinoma
}

\section{Britney $\mathrm{He}$}

Department of Oncology, Georgetown University Medical Center, Washington D.C.

E-mail: bkh40@georgetown.edu

https://doi.org/10.48091/gsr.v1i2.21

\begin{abstract}
One of the largest hurdles to the efficacy of cancer therapeutics, and a main cause of relapse, is therapy resistance. In response, researchers have developed model systems to better understand therapy resistance. Cancer research employs several model systems that reflect the biology of actual human tumors: in vitro models (2D, 3D cell cultures), in vivo models (PDX, GEMMS, transgenic), proteomic models, and computational or mathematical models. One cancer that has been extensively modeled is pancreatic ductal adenocarcinoma (PDAC). PDAC is the third most common cause of annual cancer deaths in developed countries; as its incidence and mortality rates continue to increase, PDAC is projected to be the second leading cause of cancer deaths by 2030. Although chemotherapy is a pillar of clinical PDAC treatment, its outcome typically leads to multi-drug resistance, drastically restricting the curative effect of drugs for a variety of tumors. Elucidating the underlying mechanisms for resistance through different models is essential for the development of new strategies and therapies. This review provides insight into the range of in vitro and in vivo models of pancreatic cancer used in preclinical research. This paper provides an overview of platforms for cancer research with a focus on those devoted to resistance mechanisms in PDAC and to the primary therapeutic intervention for PDAC, gemcitabine (GEM).
\end{abstract}

Keywords: therapy resistance, pancreatic cancer, in vivo models, in vitro models, computational, proteomic, gemcitabine, PDAC

\section{Introduction}

One of the most studied cancers is pancreatic ductal adenocarcinoma (PDAC) due to its high stemness and tumorigenicity, making it a major health concern that warrants greater efforts towards earlier detection and improved treatment. There are several models for PDAC on cancer resistance due to the extremely deadly nature of PDAC. Some of the newest and acclaimed models are for PDAC cells. This review will highlight the models used for examining drug resistance in PDAC.

PDAC is the third most common cause of annual cancer deaths in developed countries, and the incidence and mortality rates of this disease are climbing. This disease is projected to be the second leading cause of cancer deaths by $2030 .{ }^{67}$ Despite increased survival resulting from various multidisciplinary curative and palliative treatment options, including surgical removal, stent placement, and nonsteroidal anti-inflammatory drugs, the disease outcome is most often fatal. The most recent advances in chemotherapeutic treatment extend the average survival to 8.5-9.4 months. ${ }^{68,69}$ Currently, the 5-year survival rate for PDAC for all stages is the lowest among all the cancers at 9\%, which drops to $3 \%$ for patients diagnosed with distant or metastatic PDAC. ${ }^{70}$ The lack of effective treatment options for PDAC contributes to the low survival rate, pointing to the 
need for improved treatment and earlier detection. ${ }^{67}$

Medical and surgical treatments for this highly lethal disease are limited and often ineffective. For patients with unresectable PDAC, therapy is restricted to chemotherapy, for which most eventually develop resistance. Gemcitabine (GEM) emerged in 1997 as an alternative for 5Fluorouracil and ultimately improved survival by a few weeks. The introduction of the FOLFIRINOX treatment scheme (5-fluorouracil, leucovorin, oxaliplatin, and irinotecan) also contributed to a small improvement in survival for patients with an advantaged stage of the disease. ${ }^{55}$ Currently, the most effective and tolerated drug is nab-paclitaxel (n-PTX) and, when used with GEM, has modestly prolonged median overall survival. $^{54,69}$

The poor clinical situation and the fact that only three improvements have been introduced over the last 20 years underscore how effective therapeutic strategies for patients with PDAC have been difficult to identify. Despite numerous preclinical investigations and clinical trials, only moderate progress has been made in improving therapeutic strategies. ${ }^{55}$ Thus, there is a desperate need for novel drugs, improved radiation protocols, and increased avenues for second- and third-line therapies. ${ }^{55}$

Although chemotherapy is one of the pillars of clinical cancer treatment, its outcome typically leads to multidrug resistance, drastically restricting the curative effect of drugs for a variety of tumors, such as in pancreatic cancer patients. ${ }^{7,71}$ Many cancer types that are initially susceptible to treatment often develop therapeutic resistance over the course of the therapeutic regimen. Resistance is due to several intracellular factors, including genetic and epigenetic changes in signaling pathways, drug-metabolizing enzymes, and drug efflux pump mechanisms. ${ }^{7,71}$ Pancreatic tumors are especially characterized by genetic instability, intra-tumoral heterogeneity, and distinct desmoplastic stroma that makes it difficult to effectively develop therapeutic strategies for PDAC. The lack of innovative approaches to treating PDAC stems from the high degree of heterogeneity of this tumor with several different histopathological subtypes and limited knowledge on the molecular mechanisms behind tumor development and progression.

The development of chemotherapeutic resistance in cancer patients poses a major clinical problem for chemotherapeutic treatment. The elucidation of underlying mechanisms for resistance through different models is essential for the development of new strategies and therapies. ${ }^{7,71}$ Therapeutic resistance in PDAC has been explored through various cell culture and animal model systems. Cell cultures include twodimensional (2D) culture conditions and, most notably, three-dimensional (3D) culture strategies, such as organoids and spheroids. ${ }^{36} 3 \mathrm{D}$ culture models have incorporated pancreatic stellate cells (PSC) to investigate the prominent desmoplastic/stromal reaction in PDAC. ${ }^{62}$ Moreover, cells grown in 3D models showed resistance to GEM and n-PTX, drugs frequently used for PDAC treatment. ${ }^{36,62}$ Animal model systems include patient-derived xenografts (PDXs) and genetically engineered mouse models (GEMMs). ${ }^{36}$

This review provides an overview of the range of in vitro and in vivo models of pancreatic cancer that are being used in preclinical research. It considers an overview of platforms for cancer research with a focus on those devoted to resistance mechanisms in PDAC and the therapeutic intervention gemcitabine. The goals of the paper are to examine the impact of each tumor resistance model for PDAC.

\section{Background}

Therapeutic resistance mechanisms can be tumor cell-intrinsic (present before treatment), acquired during treatment by various therapyinduced adaptive responses, ${ }^{2}$ or mediated by the tumor microenvironment (TME).,4,5,6 Tumor molecular and genetic heterogeneity is the main reason for the failure of conventional cancer therapy as resistance can arise from the positive selection of a drug-resistant tumor subpopulation. ${ }^{5}$ 
The high adaptability of tumors through activation of pro-survival signaling pathways and the inactivation of downstream death signaling pathways can lead to drug resistance. ${ }^{2}$ The activation of epidermal growth factor receptor (EGFR) also serves as a resistance mechanism against chemotherapies, including treatments for pancreatic cancer, such as 5-fluorouracil, irinotecan, and nanoparticle albumin-bound paclitaxel (nab-paclitaxel). ${ }^{5}$

Mechanisms of resistance to cytotoxic and targeted chemotherapeutics include an increased rate of drug efflux, alterations in the drug target, activation of pro-survival compensatory signaling pathways, and ineffective induction of cell death. ${ }^{5}$ Cell plasticity also facilitates adaptive cellular reprogramming to drive acquired drug resistance. ${ }^{2}$

Drug-resistance mechanisms are regulated by the TME, epithelial-mesenchymal transitions (EMTs), and microRNA. ${ }^{7}$ Excellent reviews on the contents of the TME have been published previously $4,5,10,13,17,20,21$ and from these works, it is clear that the TME consists of the extracellular matrix, cancer-associated fibroblasts, immune and inflammatory cells, tumor-associated macrophages (TAM), and blood vessels that provide refuge for cancer cells from cytotoxic agents. Additionally, epithelial cells can undergo a transition to become mesenchymal cells by losing their polarized organization and tight cell-cell junctions to change cell shape and develop a fibroblast-like morphology. ${ }^{5}$ Studies have found a correlation between chemotherapeutic resistance and the EMT. ${ }^{23,24}$ Similarly, microRNAs (miRNAs), a class of small non-coding RNAs that negatively regulate genes at the post-transcriptional level, have also been shown to affect drug resistance. ${ }^{7}$ Recent studies have found a correlation between miRNA expression and resistance towards chemotherapeutic targets. ${ }^{7}$
Cancer research typically involves these drugresistance mechanisms and relies on model systems, which reflect the biology of human tumors to a certain extent. ${ }^{25}$ Models throughout cancer research history have addressed all stages of drug discovery, including target identification, toxicity, and individual patient prediction. Initial molecular biology models have increased understanding of tumor cell biology, while new model systems simulate functional processes related to the development and growth of cancer. Cell cultures, namely those derived from a cervical cancer patient Henrietta Lacks (HeLa), became the first laboratory model for cancer research in understanding tumor biology, drug identification, and drug development. ${ }^{25}$ In addition to $2 \mathrm{D}$ cell cultures, other models currently in use include in vitro conditionally reprogrammed cell (CRC) lines, 3D cell cultures, organoids, spheroids, and tumor-on-the-chip models, in vivo zebrafish models, patient-derived xenograft (PDX) mouse models, genetically engineered mouse models (GEMMs), and transgenic mouse models, as well as computational or mathematical models. Considering how mechanisms for tumor hypoxia, senescence, and cytoskeletal organization vary, a current challenge in cancer research is selecting the model that best reflects the given tumor entity. Thus, the roles of cellular senescence, dormancy in tumor formation, and therapy resistance have become increasingly important and relevant in cancer research. ${ }^{26}$ This paper will survey these topics and investigate their utility in various applications.

\section{Models in Cancer Research}

This section describes each of four categories of cancer research model systems: in vitro models (2D and 3D cell cultures), in vivo models (PDX, GEMMS, transgenic), proteomic models, and computational or mathematical models (Figure 1).

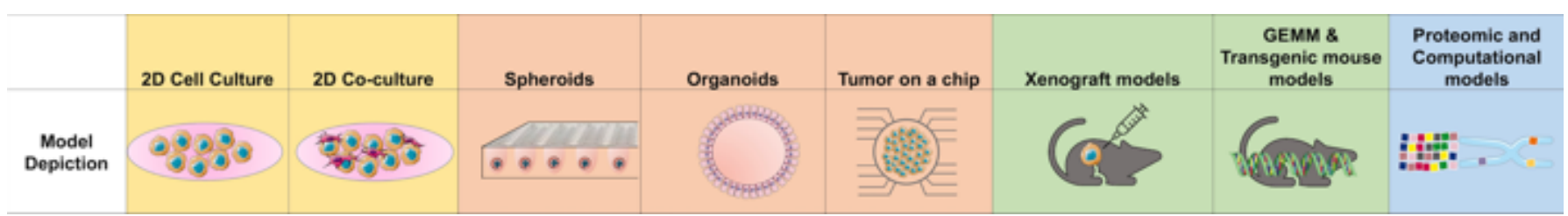

Figure 1. Models in Cancer Research 


\subsection{In Vitro Models}

\subsubsection{D Cultures}

The use of in vitro models has allowed for preclinical and translational studies of tumor mutations, aberrations, and responses to therapeutic agents. ${ }^{27} 2 \mathrm{D}$ cell culture models are inexpensive and easy to generate and maintain, making them the mainstay for cancer research. Still, these models have limitations, including induced alterations in cell morphology that translate to changes in gene and protein expression. 3D cell cultures have partially overcome this shortcoming. ${ }^{27}$

Conditionally reprogrammed cells (CRCs) overcome senescence to produce immortalized primary cell cultures through a method described by Liu et al. (the Georgetown method), which combines irradiated mouse fibroblasts as a feeder cell layer with the use of a Rho kinase (ROCK) inhibitor. ${ }^{28}$ Cells can be derived from both normal and cancer tissues and grown indefinitely under these conditions while maintaining a normal phenotype. ${ }^{25}$ The irradiated mouse fibroblasts maintain telomerase reverse transcriptase (hTERT) expression to prevent chromosome shortening and tumorigenic cellular transformation of normal cells. Furthermore, the use of ROCK inhibitors maintains the undifferentiated and proliferative state of epithelial cells. In combination with the feeder cells, ROCK inhibitors prevent the transformation or senescence of cultured cells. ${ }^{25}$ Thus, Liu et al. demonstrated that irradiated murine fibroblasts and a ROCK inhibitor are essential for both initial survival, unlimited expansion, and senescence prevention. ${ }^{28}$

Yuan et al. were one of the first to use CRCs to generate cell cultures from the patient's normal and tumorous lung tissue and study tumor progression in recurrent respiratory papillomatosis. ${ }^{29} \mathrm{By}$ co-culturing the primary cells with $\mathrm{J} 2$ murine fibroblast cells and a medium containing ROCK inhibitor Y-27632, they were able to detect a mutation of HPV-11 in the CRCs that may contribute to aggressive clinical behavior. Furthermore, the CRCs were used in screening potential drug therapies, which allowed for the identification of vorinostat as a potential therapy for patients with recurrent respiratory papillomatosis. Thus, this method of generating cell cultures from many epithelia can be used in personalized medicine and to study other cancers and diseases. CRCs have also been used in regenerative medicine, drug sensitivity testing, gene expression profiling, and xenograft studies. ${ }^{29,30,31,32}$

Recent models with CRCs include those for prostate cancer, which aimed to define potential new therapies and observe drug sensitivity and resistance. Naeem et al. modeled prostate cancer using $\mathrm{CRCs}$ of normal and prostate cancer $(\mathrm{PCa})$ cells derived from treatment-naive patients with primary $\mathrm{PCa} .{ }^{31}$ Using an integration of an in silico proteochemometric network pharmacology platform and in vitro methods with CRCs, the researchers examined drug response in sensitivity assays on $\mathrm{PCa} \mathrm{CRCs}$ and predicted novel applications for $\mathrm{PCa}$ chemotherapies, including broad applicability to rapidly identity and test approved drugs. Additionally, Tricoli et al. presented a 3D non-spheroid model using CRCs for normal and tumor-derived $\mathrm{PCa} \mathrm{CRC}$ to describe the combined effects of a multidimensional transwell platform and define culture media on $\mathrm{PCa}$ cellular proliferation, differentiation, and signaling. ${ }^{33}$ The use of a transwell-dish culture method (TDCM) enables multi-dimensional culturing of $\mathrm{PCa}$ CRCs, allowing for a more mature, stratified prostate epithelial phenotype that can be used for basic and translational studies of $\mathrm{PCa} .{ }^{34}$

\subsubsection{D Cultures}

While 2D in vitro cell culture models are widely used for studying the basic biology and tumorigenesis of various cancers, $3 \mathrm{D}$ models more accurately mimic the native cancer tissue by preserving cellular heterogeneity and replicating some of the specific biochemical and morphological features of the corresponding tissue 
in vivo. This similarity to in vivo tissue provides an advantage in the model for examining morphology, tumor microenvironment, invasion, metabolism, and cell-environment crosstalk that influences gene expression and cell behavior. Furthermore, 3D cell cultures serve as a model for experimental therapy studies using radiotherapy, chemotherapy, and cell- and antibody-based immunotherapy. ${ }^{26}$ Several reports demonstrate that cancer cells can grow in non-adherent conditions, forming 3D structures, or spheroids, that show increased resistance to drugs frequently used for cancer treatment. ${ }^{27}$ Due to their flexibility in mimicking tumor microenvironments through modifying cell culture conditions, 3D culture models are useful tools for studying cancer development and potential targets for therapeutic intervention.

The most innovative and promising approach for in vitro modeling employs tumor tissue organoids, which are self-organizing, multicellular structures derived from primary tissue and grown in well-defined conditions. Several methods for developing organoid lines and biobanks have been used for prostate cancer, ${ }^{35}$ pancreatic cancer, ${ }^{36}$ and colorectal cancer ${ }^{37}$ for in vitro drug testing. While drug sensitivity is usually exploited using rapid in vitro screening through a short-term culture of tumor sections and in vivo screening through xenotransplantation of the tumor into immunodeficient mice, organoid technology bridges these two approaches to produce a feasible medium-throughput drug screen on patientderived organoids (PDOs). ${ }^{35,37}$ These 3D organoids can maintain their complex architecture and reproduce their marker expression, allowing several research groups to study tumor development and test for drug efficacy. ${ }^{6,27,36,37,38,39,40}$ Organoid cultures typically use Matrigel or collagen and support the growth of both normal and cancer tissue such that cultures can be manipulated at the genetic level by transfection. ${ }^{27}$

When cultured with stroma and fibroblasts, organoids have the potential to simulate the full spectrum of patient cancer progression and study normal cells, preinvasive carcinomas, and metastatic cells. ${ }^{6,41,42}$ Furthermore, PDO cocultures with immune cells, ${ }^{43,44}$ cancer-associated fibroblasts, ${ }^{45}$ and stellate cells ${ }^{42}$ can identify tumor microenvironment characteristics and determine mechanisms for resistance to traditional and investigational drugs. ${ }^{6}$

Organoids are generated rapidly and reliably, especially with endoscopic ultrasound (EUS)guided tissue acquisition at the time of initial diagnosis, ${ }^{41}$ making them more usable for patients who need targeted treatment as quickly as possible. The development of PDO biobanks also greatly expands the type of patient samples that can be propagated and studied, and these samples can then be used to accurately predict drug responses in a personalized treatment setting. ${ }^{6}$

Tumor- or cancer-on-a-chip (CoC) models have been used more recently to dissect the role of tumor microenvironment cues and their role in metastasis. ${ }^{22}$ These microfluidic chip device models enable control over local gradients, fluid flow, ${ }^{46}$ tissue mechanisms ${ }^{111}$, and composition of the local environment through micrometer- to millimeter-sized compartments and microchannels. $^{22}$ The small chamber for cell culture creates a niche in which tumors can grow, develop, and interact in a specified microenvironment. ${ }^{22}$

By making it possible to manipulate the variables listed above, $\mathrm{CoC}$ models overcome some of the limitations of $2 \mathrm{D}$ or $3 \mathrm{D}$ cell cultures and animal models. The $\mathrm{CoC}$ model used depends upon the particular tumor microenvironment cues that researchers aim to understand. The many different types of $\mathrm{CoC}$ models include 2D chips, lumen chips for $\mathrm{CAFs},{ }^{47}$ compartmentalized chips for TAMs, ${ }^{48,49,50} \mathrm{CAFs},{ }^{32,51} \mathrm{Y}$ chips for $\mathrm{CAFs},{ }^{52}$ and membrane chips. Many models have a gelfluid interface lined with endothelial cells. ${ }^{48,53} \mathrm{CoC}$ models have helped better understand the invasion-related interactions between CAFs and cancer cells, as well as how activation of TAMs enhances cancer invasion through live observation of microenvironmental dynamics. 


\subsection{In Vivo Models}

An in vivo model, zebrafish, serves as an intermediate of cell culture models and mammalian models. This zebrafish model allows for extracorporeal fertilization and has a wellresearched translucent embryo, short generation time, as well as a well-developed genetical toolbox, which makes the zebrafish ideal for studying development. ${ }^{26}$ Zebrafish melanoma models have shown that the cause of cancer formation is the dedifferentiation of epithelial cells to form embryonic neural crest cells. ${ }^{26}$ Moreover, zebrafish xenograft models can also be used to determine the tumorforming capacity of PDAC CRCs and assess whether chemotherapeutic resistance was retained in vivo. ${ }^{54}$

Therapy-resistance in vivo animal models mainly use mice due to optimal short generation time (10 weeks), average life expectancy (2.5 years), the possibilities for reverse genetics, and the frequent occurrence of cancer in the absence of oncogenic agents. The use of mouse models dates to the late 1960s and the development of immunedeficient mouse strains. ${ }^{26}$

Patient-derived xenotransplants (PDX) use chemotherapy-naive tissue obtained from surgery or biopsies and transplant them into immunedeficient mouse strains, like nude or severe combined immunodeficiency (SCID). ${ }^{26,55,56,57,58,59,6}$ Immunocompetent and immunodeficient mice with xenografted tumors are traditionally transplanted subcutaneously or orthotopically..$^{61,62}$ These models are widely used due to their availability, low cost, and ability to mimic attributes of human malignancies by recapitulating neoplastic cell architecture and conserving genetic and phenotypic biology at the histological and molecular models..$^{55}$ This makes PDX models the favored method of identifying drugs that significantly inhibit tumor growth, validating tumor biomarkers, and predicting treatment outcomes. ${ }^{55}$

Highly sophisticated transgenic mouse models allow researchers to look at the early stages of tumor development and constitutively or conditionally induce the expression of an oncogenic mutation at a specific time and in a specific organ using conventional methods, such as retroviral infection, microinjection of DNA constructs, and the "gene-targeted transgene" approach. ${ }^{26,63,64}$ The use of transgenic models has been important in studies evaluating the development of resistance to therapy. ${ }^{63}$ Knockout transgenic mice, in which the gene is depleted or silenced to cause a loss of gene function, are a powerful tool for assessing the potential validity of a targeted therapy because the targets can be precisely inactivated in the developing or developed tumor. ${ }^{63}$ Recently, the use of CRISPR/Cas9-based transgenic models has allowed for more effective systems to study human cancers. ${ }^{63}$ Due to advances in immunotherapy that have illuminated the importance of immune response in tumor progression and treatment, new PDX models, namely those that interact with the human immune system, are necessary.

Humanized mouse xenograft models replace the mouse immune system with a human immune system. These models transplant CD34+ human hematopoietic stem cells (HSCs) into mice to produce human blood cells. They are potentially valuable models for new immunotherapies because they mimic tumor heterogeneity, the tumor microenvironment, and crosstalk between the tumor and stromal/immune cells. ${ }^{63}$

Wang et al. developed human hematopoietic and immune systems in mice transplanted with human (h)CD34+ hematopoietic progenitor and stem cells. ${ }^{65}$ After implanting the PDX of nonsmall cell lung cancer (NSCLC), sarcoma, bladder, cancer, and triple-negative breast cancer into the humanized NSG (huNSG) mice, the researchers discovered that tumor growth curves in the humanized mice were similar to those in nonhuman immune cell-engrafted NSG mice. Additionally, treatment with pembrolizumab, which targets programmed cell death protein 1 , produced significant growth inhibition in PDX tumors in huNSG mice but not in NSG mice. These results suggest that tumor-bearing huNSG mice can serve as a novel platform for testing the efficacy of immunotherapies. Future data collected 
from these humanized mouse models will enable the development of predictive cancer biomarkers of response to chemotherapies. Chang et al. described a novel orthotopic renal cell carcinoma (RCC) xenograft humanized mouse model as an improved model to evaluate in vivo anti-tumor capabilities of fully human monoclonal antibodies for RCC therapy ${ }^{66,55}$

Genetically engineered mouse models (GEMM), which are produced by modifying specific genes associated with cancer, provide an authentic, bridging model to patients, as the tumors created are aggressive, heterogeneous, and stromal (desmoplastic) in nature..$^{55}$ In addition to sharing similar genetic, phenotypic, and physiological characteristics with humans, GEMMs also suffer from typical cancer symptoms (bodyweight loss, cachexia, etc.) and the spontaneous formation of distant metastasis. ${ }^{55}$ The development of these symptoms makes it possible to simulate different stages of tumorigenesis.

\subsection{Computational Models}

Therapy resistance has also been studied through computational, theoretical, and mathematical models. The possibility of using bioinformatics models to create personalized medicine applies datasets to tumor material, the genome of the cancer patient, and metabolic pathways. Mathematical models based on partial differential equations often deal with the growth of cancer cell lines in vitro while considering parameters like initial cell density and concentrations of cell cycle inhibitors. ${ }^{26}$ These simulations can predict the conditions in which the tumor cells will die out.

\subsection{Gemcitabine Resistance and PDAC models}

GEM is a nucleoside analog used in chemotherapy for non-small cell lung, pancreatic, bladder, and breast cancers. ${ }^{72}$ Since its approval by the FDA in 1996, GEM has been used as a firstline treatment for patients with locally advanced (nonresectable Stage I/II) or metastatic (Stage IV) PDAC and remains the first-choice treatment for PDAC. GEM inhibits DNA synthesis, acting as an analog of cytidine to prevent chain elongation, and further induces apoptosis in cancer cells via caspase signaling. ${ }^{73}$ However, GEM treatment resistance along with the poor pharmacokinetic profile of GEM (8-12 mins in humans due to rapid metabolism) has resulted in poor treatment outcomes and drug resistance development over time. ${ }^{71,74}$

\section{Gemcitabine Resistance and In Vitro Models}

\subsection{Gemcitabine Resistance and 2D Cultures}

Panc-1, MiaPaCa-2, SW1990, and Capan-2 are $2 \mathrm{D}$ cell cultures that remain platforms used to study GEM resistance mechanisms and improve the efficacy of GEM in combination with other therapies. ${ }^{55,72}$ These cancer cells have the capability to generate high-fidelity in vitro models to explore the efficacy of anticancer drugs. Pancreatic cell lines (Capan-1, T3M4, MiaPaCa-2) with acquired GEM resistance (GEM-R) have elucidated resistance mechanisms that include signaling crosstalk to increases glucose uptake ${ }^{75}$ and kinase inhibitors capable of inhibiting the growth of GEM-resistant $\mathrm{MiaPaCa}-2$ cells. $^{76}$

Affram et al. investigated the cytotoxic effects of an alternative drug delivery system in the form of GEM-loaded solid lipid nanoparticle (GEMSLN) on patient-derived primary pancreatic cell lines (PPCL-46) and $\mathrm{MiaPaCa}-2$ pancreatic cancer cells. ${ }^{72}$ Solid lipid nanoparticles (SLNs) are nanocarriers that can be used as an alternative drug delivery system to improve therapeutic effectiveness for drugs, like GEM, with a short half-life that requires continuous parenteral administration. The researchers' cytotoxicity studies found a greater cytotoxic effect of GEMSLN treated PPCL-46 than of GEM hydrochloride (GEM-HCl) treated PPCL-46 cultures. A similar trend of higher GEM-SLN inhibition was found in $\mathrm{MiaPaCa}-2$ cultures as well. These results indicate the potential for enhanced GEM delivery and improved anticancer activity through GEM-SLN. Additionally, GEM-SLN is effective on both PPCL-46 cultures and established commercially available cell lines, 
indicating moderate effectiveness in addressing the heterogeneity of pancreatic cancer cells.

\subsubsection{Gemcitabine Resistance and 2D Co-Cultures}

Pancreatic stellate cells (PSC) are often used in co-culture experiments with PDAC cells to illuminate microenvironmental issues and provide a more accurate model than a single epithelial monolayer model. ${ }^{77,78,79,80}$ PDAC often displays a dense desmoplastic stroma, which has been associated with chemoresistance and inhibition of drug penetration. ${ }^{81} 3 \mathrm{D}$ matrices of PSC cells stimulate life-like settings and studies on these matrices reveal that additional tumor stroma components (fibroblasts, macrophages, immune cells, and endothelial cells) play an important role in therapy resistance. ${ }^{55}$

Karnevi et al. highlighted the role of PSCs in the epithelial-mesenchymal transition (EMT) as an intrinsic part of cancer progression that downregulates epithelial phenotype and cell-cell adhesions. ${ }^{24} \mathrm{Co}$-cultures of immortalized primary PSCs with Panc-1, MiaPaCa-2, and $\mathrm{BxPC}-3$ revealed down-regulated $\mathrm{E}$-cadherin levels and increased expression of vimentin, both of which indicate the role of PSCs in modulating the epithelial-mesenchymal transition (EMT).

More recently, Xiao et al. found increased expression of Yes-associated protein 1 (YAP1), a protein known to induce cancer-associated fibroblast activation in liver and breast tissues, in PSCs. ${ }^{82} \mathrm{~A}$ co-culture with $\mathrm{MiaPaCa}-2$ and human PSCs from residual surgical specimens revealed that YAP1 may play a critical role in the regulation of PSC activation, indicating a novel rationale for targeting YAP1 to reprogram the PDAC microenvironment. ${ }^{82}$ In addition to YAP1, it is speculated that secreted protein acidic and cysteine-rich (SPARC), a matricellular glycoprotein used in ECM assembly and cellmatrix communication during tumor progression, may also be related to PSC activation. High levels of SPARC expression in stromal cells indicated poor prognosis of PDAC patients and acted as a negative predictive biomarker in patients treated with GEM based chemotherapy. ${ }^{81}$
New cell lines, of human and murine origin, are continually being established and characterized for use in screening novel drug candidates and elucidating signaling pathways or (epi)genetic events involved in tumor development, progression, and the outcomes of therapy. ${ }^{55}$

\subsubsection{Gemcitabine Resistance and Conditionally Reprogrammed Cell Lines}

Studies on PDAC CRCs have been investigated with nab-paclitaxel, but not GEM. ${ }^{34}$ Drug sensitivity screens were conducted for cultures of muscle-invasive bladder cancer that revealed sensitivity to GEM. These results showed that CRCs are a feasible platform for personalized drug sensitivity testing for bladder cancer. ${ }^{83}$

\subsection{Gemcitabine Resistance and 3D models}

The application of $3 \mathrm{D}$ models has been a growing trend in PDAC studies, especially with developing models for drug screening. Oftentimes, chemotherapeutics that were effective in $2 \mathrm{D}$ models do not remain effective in 3D models. Different $\mathrm{IC}_{50}$ values between $2 \mathrm{D}$ and $3 \mathrm{D}$ models point to the clear discrepancy between the commonly used 2D drug screening versus the more complex 3D and co-culture models. Furthermore, 2D cell culture is known to not fully recapitulate tumor biology. ${ }^{73} 3 \mathrm{D}$ culture models better reflect actual tumor drug responses and aid in the identification of novel compounds that are more effective. $^{76}$ Differences between pancreatic cell lines in the morphology of both $2 \mathrm{D}$ and $3 \mathrm{D}$ cultures can be attributed to differences in origination site. $\mathrm{BxPC}-3$ obtained from PDAC lacks metastatic potential, while $\mathrm{MiaPaCa}-2$ and PANC-1, both of which are also derived from PDAC, demonstrate a predisposition to metastasis. ${ }^{84} \mathrm{COLO}-357$ and AsPC are obtained from metastatic sites, while T3M4 cells are derived from the lymph node metastatic mass and resemble $\mathrm{BxPC}-3 .^{84}$

Many models are based on co-cultures with other cell types or cells believed to contribute to the transformed phenotype and invasiveness of the 
cells. Notably, stromal-tumor cell interactions are actively studied in PDAC drug resistance. ${ }^{72}$ Recent research considers the tumor microenvironment that has been associated with metastatic progression and vascularization. These complex systems are easier to model using 3D structures, such as multicellular tumor spheroids (MCTS) ${ }^{84}$ and organoids. ${ }^{6}$ While spheroids and organoids both form 3D models, they differ in their morphology. Derived from tissue or cancer stem cells, spheroids grow in a minimum serum-free medium. They trigger an oxygen and nutrient gradient that leads to massive cell death in the center of the structure, which is why they cannot form tissue-like structures. In contrast, organoids require stem cell niche factors and extracellular matrices, which allow the organoids to differentiate and self-organize. Additionally, organoids do not exhibit a hypoxic or nutrient gradient. ${ }^{85}$

\subsubsection{Gemcitabine Resistance and Tumor Spheroids}

MCTS serve as models of PDAC tumors superior to flat cell monolayers (2D cultures) due to different geometry that leads to changes in nutrient and oxygen turnover as well as cell crosstalk. Current MCTS models are derived from several epithelial cell lines, including AsPC-1, BxPC-3, Capan-1, MiaPaCa-2, and PANC-1. ${ }^{84}$ Svirshchevskaya et al. identified three types of MCTS: while Type I, BxPC-3, and T3M4 formed a small number of large and dense spheroids, Type II, COLO-357, and AsPC-1, generated by $\mathrm{E}$-cadherin contacts, formed multiple and loose MCTS of different sizes ${ }^{84}$. Type III, MiaPaCa-2, and PANC-1 cells grew as floating monolayer films as they were unable to form MCTS. Cell growth for these 3D cultures and monolayers revealed a dramatic (2 order) reduction in cell proliferation for 3D type I cell line cultures treated with GEM. Drug resistance in the type I 3D cultures was found to be associated with a quiescent state (decreased proliferation) and a high level of spontaneous apoptosis in cells. Meanwhile, type II and III MCTS had comparable sensitivity to the antitumor drugs.
Ware et al. describe the generation of a 3D PDAC in vitro micro-tumor model that encompasses a stromal component using PSCs, which are myofibroblast-like cells located in the exocrine areas of the pancreas. ${ }^{2}{ }^{2}$ PSCs play a role in normal pancreatic architecture as they secrete extracellular matrix (ECM) components and are the principal source of fibrosis in the stroma. Additionally, sequestration of chemotherapeutic agents, such as GEM, occurs in the tumor stroma, effectively reducing the amount of drug that can reach cancer cells. Their PDAC stroma spheroids model presented decreased cytostaticity of GEM when compared with spheroids grown without PSCs. A study by Lee et al. corroborates these findings. ${ }^{86}$ This model will allow for improved knowledge of PDAC biology and can be used to investigate pathways that can be therapeutically targeted to inhibit PSC activation and subsequent development of fibrosis in PDAC.

\subsubsection{Gemcitabine Resistance and Organoids}

Pancreatic ductal organoids are ex-vivo models that can be established using very small biopsies, such as fine-needle aspirates 36,41 and allow for the study of localized, advanced, and metastatic patients. Boj et al. established organoid models from normal and malignant murine and human pancreas tissues to investigate the pathogenesis and address the deficiency in a comprehensive $3 \mathrm{D}$ cell culture model of murine and human PDAC progression. ${ }^{36}$ This model was used to identify genetic drivers, therapeutic targets, diagnostics, and progression for PDAC.

Tiriac et al. used EUS fine-needle biopsy (EUS-FNB) sampling to rapidly establish human PDAC organoids within 2 weeks of the EUS procedure and assessed the feasibility of this model in creating personalized treatment strategies at the time of initial tumor diagnosis and over the course of a patient's treatment. PDAC patients are ideal for EUS-FNB derived organoids as most patients will not undergo surgery, all patients need a tissue diagnosis before therapies are initiated, and only a small amount of tissue is needed for organoid creation. Successful organoid generation is 
necessary for developing personalized medicine platforms for PDAC patients. ${ }^{41}$

These 3D primary ex vivo culture systems model a spectrum of tumor stages and have elucidated important disease progression findings. Profiling with next-generation sequencing of DNA and RNA in combination with pharmacotyping can be used to predict responses in PDAC patients and provide a pathway for prioritizing therapy. ${ }^{87}$

\subsubsection{Gemcitabine Resistance and Tumor-on-a-chip}

To overcome limitations of traditional disease model systems, organ- or tumor-on-a-chip systems aim to fully recapitulate the physiology and microenvironment of tissues through spatial and fluid control of tissue architecture.

Kramer et al. examined the effect of interstitial flow on GEM resistance in PDAC using a 3D microfluidic platform. Interstitial pressure and flow are hallmarks of PDAC pathogenesis. ${ }^{73}$ The study used 3D cultures of S2-028 cells, a nonmetastatic pancreatic cancer line, and found an increase in mRNA expression of 5 multidrug resistance proteins (MRPs) when the PDAC cells were subjected to interstitial flow. This flowinduced MRP expression hints towards another factor that contributes to GEM resistance via elevation of drug efflux transport.

\section{Gemcitabine Resistance and In Vivo Models}

\subsection{Gemcitabine Resistance and Xenograft Models}

Cell line-derived xenograft models, such as $\mathrm{BxPC}-3$, MiaPaCa-2, and $\mathrm{Panc}-1$ xenografts, are popular for drug screening and resistance studies in PDAC that can be applied to increasing patient survival. Novel tumor growth-inhibiting compounds derived from gemcitabine have been applied to Panc- 1 and $\mathrm{MiaPaCa}-2$ bearing mice. ${ }^{88}$

Xenograft tumor assays have demonstrated that the tetracyclic diterpenoid compound Ordonin overcomes gemcitabine resistance in gemcitabine-resistant Panc-1 cells (PANC$1 / \mathrm{GEM}$ ) through suppressing tumorigenicity in nude mice. Additionally, a combination treatment of oridonin and gemcitabine decreased tumor growth. ${ }^{88}$ Gemcitabine resistance is mediated by a special AT-rich sequence binding protein 1 (SATB-1), a chromatin organizer that is secreted by cancer-associated fibroblasts (CAFs) ${ }^{89}$ SATB1 plays a vital role in the proliferation capacity of SW1990 tumor cells in mouse xenograft models. SATB-1 has been associated with poor prognosis and tumorigenesis in pancreatic cancer. ${ }^{89,90,91}$

Xenograft models have also revealed the role of Prolactin receptors in suppressing tumor growth. Dandawate et al. studied the role of prolactin receptors in PDAC through the injection of a diphenylbutylpiperidine-class antipsychotic drug, penfluridol, which binds to prolactin receptors. ${ }^{92}$ Penfluridol slowed the growth and volume of tumors in Panc-1 xenografts in athymic nude mice and PDX in immunodeficient NSG. Western blot analyses suggested that penfluridol induces autophagy-related proteins p62, ATG-5, ATG-7, ATG-12, LC3B, and beclin-1 to suppress PDAC tumor growth. ${ }^{92}$ Additionally, cysteine transport, $\mathrm{xCT}$, is key to tumor growth. Tumor xenograft growth of genomic knockouts of $\mathrm{xCT}$ was delayed but not suppressed, indicating the key role of $\mathrm{xCT}$ and the presence of additional mechanisms for cysteine homeostasis in vivo. ${ }^{93}$

Patient-derived xenograft (PDX) models are widely used for various solid tumors. Typically, chemotherapy-naive tumor tissue obtained from surgery or biopsies is transplanted directly into immuno-deficient mice. ${ }^{55}$ PDX models for pancreatic cancer are used to identify drugs that significantly inhibit tumor growth or to validate prognostic biomarkers, as these models are highly representative of their respective tumors due to a high degree of genetic stability observed by short tandem repeat (STR) profiling and mutation analysis. ${ }^{94}$ Well-defined PDX collections can be used to associate biomarkers with drug sensitivity and resistance to facilitate precision cancer medicine. Most publications on pancreatic PDX models describe the establishment, characterization, and preclinical application of 
PDXs, but they have yet to be applied towards clinical studies.

Tang et al. used GEM treated PDX models to show that $\mathrm{m}^{6 \mathrm{~A}}$ demethylase ALKBH5 is downregulated. Overexpression of this demethylase sensitizes PDAC cells to chemotherapy. Thus, lower levels of ALKBH5 predict poor clinical outcomes in PDAC and other cancers. ${ }^{95}$ Wei et al. used in vivo CRISPR gene knockout screening in PDX mice to identify effective lethal drug combinations that synergize with GEM for treating PDAC. ${ }^{96}$ Using a clinically relevant PDX model of PDAC with a patient tumor being propagated within the pancreas of athymic nude mice, they screened for chromatin regulators whose depletion may create conditional lethality with GEM. They found that inhibition of the protein PRMT5 led to synergistic vulnerability of PDAC cells to GEM. PRMT5 has been considered as a critical driver of cancer progression for multiple advanced-stage cancers. This study suggests that GEM treatment combined with inhibition of PRMT5 will have stronger effects and selectivity towards PDAC. ${ }^{96}$

\subsection{Gemcitabine Resistance and Genetically Engineered/Transgenic Models}

A major drawback of PDX models is the use of immunodeficient mice that lack a competent immune system to investigate immunotherapeutics. Tumor cells used in PDX have also been passaged extensively in vitro, limiting tumor cell heterogeneity and potential biological relevance. Thus, genetically engineered mice that spontaneously develop PDAC are appealing for drug discovery, especially since tumors arise in competent and fully intact immune systems.

GEMMs are similar to humans in terms of genetic, phenotypic, and physiological characteristics. Models include KC (ras ${ }^{\text {LSL.G12D/+ }}$

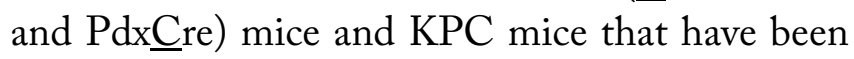
used to investigate the influence of Kras. ${ }^{55} \mathrm{KC}$ mice have normal pancreatic organogenesis and develop intraepithelial neoplasia (PanIN) that eventually progress to PDAC. KPC ( Kras $^{\text {LSL.G12D/+ }}$, p53 $3^{\mathrm{R} 172 \mathrm{H} /}+$, and $\mathrm{Pdx} \underline{\mathrm{Cre}}$ ) mice have a conditional expression of the $\mathrm{R} 72 \mathrm{H}$ mutation in the $\mathrm{p} 53$ gene in the $\mathrm{Kras}^{\mathrm{G} 12 \mathrm{D}}$ context. $\mathrm{KPC}$ is the most extensively studied genetic model of PDAC for the evaluation of immunotherapy. ${ }^{97}$ At least 40 GEMM have been generated for analysis of gene function in PDAC. ${ }^{98,99}$

Tadros et al. observed an increase in fatty acid synthase expression that corresponded with increased disease progression in PDAC GEMMs. ${ }^{100}$ Based on analysis and identification of the lipid metabolism pathway to be the most significantly enriched in tumors from patients with PDAC, they manipulated the fatty acid biosynthesis pathway. Fatty acid synthesis is also regulated by multiple transcriptional regulators, including c-MYC, which is significantly amplified in PDAC..$^{54}$ Through treatment with orlistat, the researchers demonstrated a way to overcome GEM resistance in pancreatic cancer by regulating endoplasmic reticulum stress and stemness.

Buccholz et al. showed that depletion of pharmacological tumor-associated macrophages (TAMs) improves therapeutic response to GEM in KPC mice. ${ }^{101}$ Macrophages are abundant in fibro-inflammatory TME of KC and KPC mice, which has been shown to correlate with a worse prognosis in PDAC. ${ }^{102}$ Following enrollment of KPC mice that had developed pancreatic tumors with liposomal clodronate to deplete intratumoral TAMS and GEM, they demonstrated improved efficacy of GEM in the KPC. These results point to another instance of the role of the TME in GEM resistance.

Principe et al. evaluated the effects of prolonged GEM treatment using KPC mice and found increased $\mathrm{CCL} / \mathrm{CXCL}$ cytokine/chemokine secretion and upregulation of immune surface proteins, including transforming growth factor $\beta$ (TGF $\beta$ )-associated signals, in the tumor stroma. ${ }^{103}$ TGF $\beta$-associated signals confer drug-resistant phenotypes to neighboring stromal cells and further enhance the production of inflammatory cytokines/chemokines.

Halbrook et al. examined how tumorassociated macrophages (TAMs) drive resistance 
to GEM in PDAC cell lines. They found that TAMs release a spectrum of pyrimidine species, such as deoxycytidine, that inhibit GEM through direct competition, hindering drug efficacy. KPC mice treated with GEM combination treatments had prolonged survival compared to control mice, indicating that inhibiting macrophage recruitment has the potential to improve current PDAC therapies, as seen with FOLFIRINOX. ${ }^{104}$

Özdemir et al. used transgenic (Ptf1acre/+;LSL-KrasG12D/+;Tgfbr2flox/flox)

mice with deleted $\alpha \mathrm{SMA}+$ myofibroblasts in pancreatic cancer. Myofibroblast depleted tumors did not respond to GEM and resulted in multiple adverse outcomes. Their results suggest that fibrosis associated with myofibroblasts and type I collagen constitutes a protective response from the host rather than offering an oncogenic supportive role. ${ }^{105}$

\section{Gemcitabine Resistance and Proteomic and Computational Models}

GEM resistance has also been investigated using proteomic and computational models. While biological processes of drug resistance have been described before, proteomics serves as a powerful tool for better understanding molecular mechanisms of GEM resistance. ${ }^{106}$ Proteomics investigates proteins whose expressions differ between drug-sensitive and drug-resistance cells. This method can provide system-wide views of signaling networks to better understand drug mechanisms of actions and interactions. ${ }^{106}$ Proteomics also provides the knowledge needed to identify biomarkers and for targeting specific protein pathways. ${ }^{106}$

Chen et al. examined mechanisms associated with GEM-induced resistance using 2D-DIGE and MALDI-TOF mass spectrometry and compared the proteomic alternations of a panel of differential GEM-resistant PANC-1 cells and GEM-sensitive pancreatic cells. They found that 33 proteins were differentially expressed between GEM-sensitive and GEM-resistant cells. ${ }^{107}$
Zhu et al. studied GEM with birinapant in PDAC. They identified 4069 drug-responsive proteins and quantified them in a time-series proteome analysis to highlight and quantify signaling pathways. Pathways related to DNA damage response, DNA repair, anti-apoptosis, pro-migration/invasion were implicated as underlying mechanisms for gemcitabine resistance. This study identified promising drug targets for future investigation. ${ }^{106}$

Law et al. analyzed clinical PDAC liver metastases with quantitative proteomics. Their proteomic analysis of molecular signatures unique to the disease subtypes identified GEM-induced alterations in proteins, such as serine hydroxymethyltransferase 1 , that are associated with drug resistance. ${ }^{108}$ PDAC subtypes can be characterized using proteomics and can be used to inform first-line cancer treatment. These efforts illustrate the potential of applying proteomics to improve PDAC subtype classification and therefore early detection and treatment of PDAC.

Computational modeling of gemcitabinebased therapies has also been conducted to determine optimal intervention strategies. ${ }^{109}$ Furthermore, transcriptomics has been implemented to better under the tumor microenvironment and chart changes in the fibroblastic landscape in PDAC progression. ${ }^{110}$

\section{Conclusion}

Models are essential to addressing issues of drug resistance in cancer phenotypes. While GEM resistance in PDAC continues to result in dire outcomes for patients, models can serve as a step towards elucidating resistance mechanisms to improve treatment protocols. The number of models currently in use can reproduce a wide range of tumor mechanisms to ultimately understand factors such as cell-cell interactions and the tumor microenvironment. The use of various in vivo, in vitro, proteomic, and computational models will be crucial in making a clinical impact and 
benefiting PDAC patients with better platforms for treatment and diagnosis.

\section{References}

1. Kroemer, G., \& Pouyssegur, J. (2008). Tumor cell metabolism: Cancer's achilles' heel. Cancer Cell, 13(6), 472-482.

https://doi.org/10.1016/j.ccr.2008.05.005

2. Hammerlindl, H., \& Schaider, H. (2018). Tumor cell-intrinsic phenotypic plasticity facilitates adaptive cellular reprogramming driving acquired drug resistance. Journal of Cell Communication and Signaling, 12(1), 133-141.

https://doi.org/10.1007/s12079-017-0435-1

3. Kelderman, S., Schumacher, T. N. M., \& Haanen, John B. A. G. (2015). ScienceDirect. Clinical Microbiology Newsletter, 37(4), 33.

https://doi.org/10.1016/j.clinmicnews.2015.01.008

4. Ireland, L., Santos, A., Ahmed, M. S., Rainer, C., Nielsen, S. R., Quaranta, V., et al. (2016).

Chemoresistance in pancreatic cancer is driven by stroma-derived insulin-like growth factors. Cancer Research, 76(23), 6851-6863.

5. Holohan, C., Van Schaeybroeck, S., Longley, D. B., \& Johnston, P. G. (2013). Cancer drug resistance: An evolving paradigm. Nature Reviews. Cancer, 13(10), 714-726. https://doi.org/10.1038/nrc3599

6. Tuveson, D., \& Clevers, H. (2019). Cancer modeling meets human organoid technology. Science (American Association for the Advancement of Science), 364(6444), 952-955.

https://doi.org/10.1126/science.aaw6985

7. Zeng, Pöttler, Lan, Grützmann, Pilarsky, \& Yang. (2019). Chemoresistance in pancreatic cancer. International Journal of Molecular Sciences, 20(18), 4504. https://doi.org/10.3390/ijms20184504

8. Bissell, M. J., Hall, H. G., \& Parry, G. (1982). How does the extracellular matrix direct gene expression? Journal of Theoretical Biology, 99(1), 3168. https://doi.org/0022-5193(82)90388-5

9. Lu, P., Weaver, V. M., \&Werb, Z. (2012). The extracellular matrix: A dynamic niche in cancer progression. The Journal of Cell Biology, 196(4), 395-406. https://doi.org/10.1083/jcb.201102147
10. Butcher, D. T., Alliston, T., \& Weaver, V. M. (2009). A tense situation: Forcing tumour progression. Nature Reviews. Cancer, 9(2), 108122. https://doi.org/10.1038/nrc2544

11. Kumar, S., \& Weaver, V. M. (2009). Mechanics, malignancy, and metastasis: The force journey of a tumor cell. Cancer Metastasis Reviews, 28(1-2), 113-127. https://doi.org/10.1007/s10555-0089173-4

12. Paszek, M. J., \&Weaver, V. M. (2004). The tension mounts: Mechanics meets morphogenesis and malignancy. Journal of Mammary Gland Biology and Neoplasia, 9(4), 325-342. https://doi.org/10.1007/s10911-004-1404-x

13. Xouri, G., \& Christian, S. (2010). Origin and function of tumor stroma fibroblasts. Seminars in Cell E Developmental Biology, 21(1), 40-46. https://doi.org/10.1016/j.semcdb.2009.11.017

14. Cirri, P., \& Chiarugi, P. (2012). Cancerassociated-fibroblasts and tumour cells: A diabolic liaison driving cancer progression. Cancer Metastasis Reviews, 31(1-2), 195-208. https://doi.org/10.1007/s10555-011-9340-x

15. Kalluri, R., \& Zeisberg, M. (2006). Fibroblasts in cancer. Nature Reviews. Cancer, 6(5), 392-401. https://doi.org/nrc1877

16. Balkwill, F., \& Mantovani, A. (2001). Inflammation and cancer: Back to virchow? Lancet (London, England), 357(9255), 539-545. https://doi.org/10.1016/S0140-6736(00)04046-0

17. Coussens, L. M., \& Werb, Z. (2002). Inflammation and cancer. Nature, 420(6917), 860867. https://doi.org/10.1038/nature01322

18. Mantovani, A., Allavena, P., Sica, A., \& Balkwill, F. (2008). Cancer-related inflammation. Nature, 454(7203), 436-444. https://doi.org/10.1038/nature07205

19. Hu, W., Li, X., Zhang, C., Yang, Y., Jiang, J., \& Wu, C. (2016). Tumor-associated macrophages in cancers. Clinical E Translational Oncology: Official Publication of the Federation of Spanish Oncology Societies and of the National Cancer Institute of Mexico, 18(3), 251-258. https://doi.org/10.1007/s12094-015-1373-0

20. Mantovani, A., \& Sica, A. (2010). Macrophages, innate immunity and cancer: Balance, tolerance, and diversity. Current Opinion in Immunology, 
22(2), 231-237.

https://doi.org/10.1016/j.coi.2010.01.009

21. Shieh, A. C., \& Swartz, M. A. (2011). Regulation of tumor invasion by interstitial fluid flow. Physical Biology, 8(1), 015012.

https://doi.org/10.1088/1478-3975/8/1/015012

22. Sleeboom, J. J. F., Eslami Amirabadi, H., Nair, P., Sahlgren, C. M., \& den Toonder, Jaap M. J. (2018). Metastasis in context: Modeling the tumor microenvironment with cancer-on-a-chip approaches. Disease Models E Mechanisms, 11(3) https://doi.org/10.1242/dmm.033100

23. Fuchs, B. C., Fujii, T., Dorfman, J. D., Goodwin, J. M., Zhu, A. X., Lanuti, M., et al. (2008). Epithelial-to-mesenchymal transition and integrin-linked kinase mediate sensitivity to epidermal growth factor receptor inhibition in human hepatoma cells. Cancer Research, 68(7), 2391-2399.

24. Karnevi, E., Rosendahl, A. H., Hilmersson, K. S., Saleem, M. A., \& Andersson, R. (2016). Impact by pancreatic stellate cells on epithelialmesenchymal transition and pancreatic cancer cell invasion: Adding a third dimension in vitro. Experimental Cell Research, 346(2), 206-215. https://doi.org/10.1016/j.yexcr.2016.07.017

25. Agarwal, S., \& Rimm, D. L. (2012). Making every cell like HeLa: A giant step for cell culture. The American Journal of Pathology, 180(2), 443-445. https://doi.org/10.1016/j.ajpath.2011.12.001

26. Breitenbach, M., \& Hoffmann, J. (2018). Editorial: Cancer models. Frontiers in Oncology, 8 https://doi.org/10.3389/fonc.2018.00401

27. Gaebler, M., Silvestri, A., Haybaeck, J., Reichardt, P., Lowery, C. D., Stancato, L. F., et al. (2017). Three-dimensional patient-derived in vitro sarcoma models: Promising tools for improving clinical tumor management. Frontiers in Oncology, 7 https://doi.org/10.3389/fonc.2017.00203

28. Liu, X., Ory, V., Chapman, S., Yuan, H., Albanese, C., Kallakury, B., et al. (2012). ROCK inhibitor and feeder cells induce the conditional reprogramming of epithelial cells. The American Journal of Pathology, 180(2), 599-607. https://doi.org/10.1016/j.ajpath.2011.10.036

29. Yuan, H., Myers, S., Wang, J., Zhou, D., Woo, J. A., Kallakury, B., et al. (2012). Use of reprogrammed cells to identify therapy for respiratory papillomatosis. The New England Journal of Medicine, 367(13), 1220-1227. https://doi.org/10.1056/NEJMoa1203055

30. Tricoli, L., Naeem, A., Parasido, E., Mikhaiel, J. P., Choudhry, M. U., Berry, D. L., et al. (2018). Characterization of the effects of defined, multidimensional culture conditions on conditionally reprogrammed primary human prostate cells. Oncotarget, 9(2), 2193-2207. https://doi.org/10.18632/oncotarget.23363

31. Naeem, A., Dakshanamurthy, S., Walthieu, H., Parasido, E., Avantaggiati, M., Tricoli, L., et al. (2020). Predicting new drug indications for prostate cancer: The integration of an in silico proteochemometric network pharmacology platform with patient-derived primary prostate cells. The Prostate, 80(14), 1233-1243. https://doi.org.10.1002/pros.24050

32. Liu, X., Krawczyk, E., Suprynowicz, F. A., Palechor-Ceron, N., Yuan, H., Dakic, A., et al. (2017). Conditional reprogramming and long-term expansion of normal and tumor cells from human biospecimens. Nature Protocols, 12(2), 439-451. https://doi.org/10.1038/nprot.2016.174

33. Tricoli, L., Berry, D. L., \& Albanese, C. (2017). A rapid filter insert-based $3 \mathrm{D}$ culture system for primary prostate cell differentiation. Journal of Visualized Experiments, (120) https://doi.org/10.3791/55279

34. Ringer, L., Sirajuddin, P., Tricoli, L., Waye, S., Choudhry, M. U., Parasido, E., et al. (2014). The induction of the p53 tumor suppressor protein bridges the apoptotic and autophagic signaling pathways to regulate cell death in prostate cancer cells. Oncotarget, 5(21), 10678-10691.

https://doi.org/10.18632/oncotarget.2528

35. Gao, D., Vela, I., Sboner, A., Iaquinta, P. J., Karthaus, W. R., Gopalan, A., et al. (2014). Organoid cultures derived from patients with advanced prostate cancer. Cell, 159(1), 176-187. https://doi.org/10.1016/j.cell.2014.08.016

36. Boj, S. F., Hwang, C., Baker, L. A., Chio, I. I. C., Engle, D. D., Corbo, V., et al. (2015). Organoid models of human and mouse ductal pancreatic cancer. Cell, 160(1-2), 324-338.

https://doi.org/10.1016/j.cell.2014.12.021 
37. van de Wetering, M., Francies, H., Francis, J., Bounova, G., Iorio, F., Pronk, A., et al. (2015). Prospective derivation of a living organoid biobank of colorectal cancer patients. Cell (Cambridge), 161(4), 933-945. https://doi.org/10.1016/j.cell.2015.03.053

38. Sachs, N., \& Clevers, H. (2014). Organoid cultures for the analysis of cancer phenotypes. Current Opinion in Genetics \& Development, 24, 6873. https://doi.org/10.1016/j.gde.2013.11.012

39. Li, X., Nadauld, L., Ootani, A., Corney, D. C., Pai, R. K., Gevaert, O., et al. (2014). Oncogenic transformation of diverse gastrointestinal tissues in primary organoid culture. Nature Medicine, 20(7), 769-777. https://doi.org/10.1038/nm.3585

40. Nadauld, L. D., Garcia, S., Natsoulis, G., Bell, J. M., Miotke, L., Hopmans, E. S., et al. (2014). Metastatic tumor evolution and organoid modeling implicate TGFBR2 as a cancer driver in diffuse gastric cancer. Genome Biology, 15(8), 428. https://doi.org/10.1186/s13059-014-0428-9

41. Tiriac, H., Bucobo, J. C., Tzimas, D., Grewal, S., LaComb, J., Rowehl, L., et al. (2018). Successful creation of pancreatic cancer organoids by means of eus-guided fine-needle biopsy (EUS-FNB) for personalized cancer treatment. Gastrointestinal Endoscopy, 87(6), AB50-AB51. https://doi.org/10.1016/j.gie.2017.12.032

42. Öhlund, D. (2017). Distinct populations of inflammatory fibroblasts and myofibroblasts in pancreatic cancer Retrieved from http://urn.kb.se/resolve?urn=urn:nbn:se:umu:diva$\underline{132073}$

43. Neal, J. T., Li, X., Zhu, J., Giangarra, V., Grzeskowiak, C. L., Ju, J., et al. (2018). Organoid modeling of the tumor immune microenvironment Mendeley.

44. Dijkstra, K. K., Cattaneo, C. M., Weeber, F., Chalabi, M., van de Haar, J., Fanchi, L. F., et al. (2018). Generation of tumor-reactive T cells by co-culture of peripheral blood lymphocytes and tumor organoids. Cell, 174(6), 1586-1598.e12. https://doi.org/S0092-8674(18)30903-6

45. Biffi, G., Oni, T. E., Spielman, B., Hao, Y., Elyada, E., Park, Y., et al. (2019). IL1-induced JAK/STAT signaling is antagonized by TGF $\beta$ to shape CAF heterogeneity in pancreatic ductal adenocarcinoma. Cancer Discovery, 9(2), 282-301.

46. Kalchman, J., Kalchman, J., Fujioka, S., Fujioka, S., Chung, S., Chung, S., et al. (2013). A threedimensional microfluidic tumor cell migration assay to screen the effect of anti-migratory drugs and interstitial flow. Microfluidics and Nanofluidics, 14(6), 969-981. https://doi.org/10.1007/s10404012-1104-6

47. Bischel, L. L., Beebe, D. J., \& Sung, K. E. (2015). Microfluidic model of ductal carcinoma in situ with 3D, organotypic structure. BMC Cancer, 15, 12. https://doi.org/10.1186/s12885-015-1007-5

48. Zervantonakis, I. K., Hughes-Alford, S. K., Charest, J. L., Condeelis, J. S., Gertler, F. B., \& Kamm, R. D. (2012). Three-dimensional microfluidic model for tumor cell intravasation and endothelial barrier function. Proceedings of the National Academy of Sciences of the United States of America, 109(34), 13515-13520. https://doi.org/10.1073/pnas.1210182109

49. Bai, J., Adriani, G., Dang, T., Tu, T., Penny, H. L., Wong, S., et al. (2015). Contact-dependent carcinoma aggregate dispersion by $\mathrm{M} 2 \mathrm{a}$ macrophages via ICAM- 1 and $\beta 2$ integrin interactions. Oncotarget, 6(28), 25295-25307. https://doi.org/10.18632/oncotarget.4716

50. Huang, C. P., Lu, J., Seon, H., Lee, A. P., Flanagan, L. A., Kim, H., et al. (2009). Engineering microscale cellular niches for threedimensional multicellular co-cultures. Lab on a Chip, 9(12), 1740-1748. https://doi.org/10.1039/b818401a

51. Li, J., Jia, Z., Kong, J., Zhang, F., Fang, S., Li, X., et al. (2016). Carcinoma-associated fibroblasts lead the invasion of salivary gland adenoid cystic carcinoma cells by creating an invasive track. PloS One, 11(3), e0150247. https://doi.org/10.1371/journal.pone.0150247

52. Sung, K. E., Yang, N., Pehlke, C., Keely, P. J., Eliceiri, K. W., Friedl, A., et al. (2011). Transition to invasion in breast cancer: A microfluidic in vitro model enables examination of spatial and temporal effects. Integrative Biology: Quantitative Biosciences from Nano to Macro, 3(4), 439-450. https://doi.org/10.1039/c0ib00063a 
53. Wong, A. D., \& Searson, P. C. (2014). Live-cell imaging of invasion and intravasation in an artificial microvessel platform. Cancer Research, 74(17), 4937-4945. https://doi.org/10.1158/00085472.CAN-14-1042

54. Parasido, E., Avetian, G. S., Naeem, A., Graham, G., Pishvaian, M., Glasgow, E., et al. (2019). The sustained induction of c-MYC drives nabpaclitaxel resistance in primary pancreatic ductal carcinoma cells. Molecular Cancer Research, 17(9), 1815-1827. https://doi.org/10.1158/15417786.mcr-19-0191

55. Behrens, D., Walther, W., \& Fichtner, I. (2017). Pharmacology \& therapeutics. Pharmacology \& Therapeutics, 173, 146-158. Retrieved from http://www.sciencedirect.com/science/article/pii/S $016372581730027 \mathrm{X}$

56. Allen-Petersen, B. L., Risom, T., Feng, Z., Wang, Z., Jenny, Z. P., Thoma, M. C., et al. (2019). Activation of PP2A and inhibition of mTOR synergistically reduce MYC signaling and decrease tumor growth in pancreatic ductal adenocarcinoma. Cancer Research (Chicago, Ill.), 79(1), 209-219. https://doi.org/10.1158/00085472.can-18-0717

57. ter Brugge, P., Kristel, P., van der Burg, E., Boon, U., de Maaker, M., Lips, E., et al. (2016).

Mechanisms of therapy resistance in patientderived xenograft models of BRCA1-deficient breast cancer. JNCI: Journal of the National Cancer Institute, 108(11) https://doi.org/10.1093/jnci/djw148

58. Tentler, J. J., Tan, A. C., Weekes, C. D., Jimeno, A., Leong, S., Pitts, T. M., et al. (2012). Patientderived tumour xenografts as models for oncology drug development. Nature Reviews. Clinical Oncology, 9(6), 338-350. https://doi.org/10.1038/nrclinonc.2012.61

59. Boone, J. D., Dobbin, Z. C., Straughn, J. M., \& Buchsbaum, D. J. (2015). Ovarian and cervical cancer patient derived xenografts: The past, present, and future. Gynecologic Oncology, 138(2), 486-491. https://doi.org/10.1016/j.ygyno.2015.05.022

60. Bernardo, C., Costa, C., Sousa, N., Amado, F., \& Santos, L. (2015). Patient-derived bladder cancer xenografts: A systematic review. Translational
Research: The Journal of Laboratory and Clinical Medicine, 166(4), 324-331. https://doi.org/10.1016/j.trsl.2015.02.001

61. Okumura, M., Ichihara, H., \& Matsumoto, Y. (2018). Hybrid liposomes showing enhanced accumulation in tumors as theranostic agents in the orthotopic graft model mouse of colorectal cancer. Drug Delivery, 25(1), 1192-1199. https://doi.org/10.1080/10717544.2018.1475517

62. Ware, M. J., Keshishian, V., Law, J. J., Ho, J. C., Favela, C. A., Rees, P., et al. (2016). Generation of an in vitro 3D PDAC stroma rich spheroid model. Biomaterials, 108, 129-142. https://doi.org/10.1016/j.biomaterials.2016.08.041

63. Lampreht Tratar, U., Horvat, S., \& Cemazar, M. (2018). Transgenic mouse models in cancer research. Frontiers in Oncology, 8 https://doi.org/10.3389/fonc. 2018.00268

64. Ikink, G. J., Boer, M., Bakker, E. R. M., VendelZwaagstra, A., Klijn, C., ten Hoeve, J., et al. (2018). Insertional mutagenesis in a HER2positive breast cancer model reveals ERAS as a driver of cancer and therapy resistance. Oncogene, 37(12), 1594-1609. https://doi.org/10.1038/s41388-017-0031-0

65. Wang, M., Yao, L., Cheng, M., Cai, D., Martinek, J., Pan, C., et al. (2018). Humanized mice in studying efficacy and mechanisms of PD1-targeted cancer immunotherapy. The FASEB Journal, 32(3), 1537-1549. https://doi.org/10.1096/fj.201700740r

66. Chang, D., Moniz, R. J., Xu, Z., Sun, J., Signoretti, S., Zhu, Q., et al. (2015). Human antiCAIX antibodies mediate immune cell inhibition of renal cell carcinoma in vitro and in a humanized mouse model in vivo. Molecular Cancer, 14, 119. https://doi.org/10.1186/s12943-015-0384-3

67. Rahib, L., Smith, B. D., Aizenberg, R., Rosenzweig, A. B., Fleshman, J. M., \& Matrisian, L. M. (2014). Projecting cancer incidence and deaths to 2030: The unexpected burden of thyroid, liver, and pancreas cancers in the united states. Cancer Research, 74(11), 2913-2921.

68. Laquente, B., Calsina-Berna, A., CarmonaBayonas, A., Jiménez-Fonseca, P., Peiró, I., \& Carrato, A. (2017). Supportive care in pancreatic ductal adenocarcinoma. Clinical \& Translational 
Oncology, 19(11), 1293-1302.

https://doi.org/10.1007/s12094-017-1682-6

69. Blomstrand, H., Scheibling, U., Bratthäll, C., Green, H., \& Elander, N. O. (2019). Real world evidence on gemcitabine and nab-paclitaxel combination chemotherapy in advanced pancreatic cancer. BMC Cancer, 19(1), 40.

https://doi.org/10.1186/s12885-018-5244-2

70. Siegel, R. L., Miller, K. D., \& Jemal, A. (2020). Cancer statistics, 2020. CA: A Cancer Journal for Clinicians, 70(1), 7-30. https://doi.org/10.3322/caac.21590

71. Adamska, A., Domenichini, A., \& Falasca, M. (2017). Pancreatic ductal adenocarcinoma: Current and evolving therapies. International Journal of Molecular Sciences, 18(7) https://doi.org/10.3390/ijms18071338

72. Affram, K., Smith, T., Ofori, E., Krishnan, S., Underwood, P., Trevino, J., et al. (2020).

Cytotoxic effects of gemcitabine-loaded solid lipid nanoparticles in pancreatic cancer cells.55, 101374.

Retrieved from

https://www.sciencedirect.com/science/journal/177 32247

73. Kramer, B., Haan, L. d., Vermeer, M., Olivier, T., Hankemeier, T., Vulto, P., et al. (2019).

Interstitial flow recapitulates gemcitabine chemoresistance in A 3D microfluidic pancreatic ductal adenocarcinoma model by induction of multidrug resistance proteins. International Journal of Molecular Sciences, 20(18), 4647. https://doi.org/10.3390/ijms20184647

74. Chin, V., Nagrial, A., Sjoquist, K., O'Connor, C. A., Chantrill, L., Biankin, A. V., et al. (2018). Chemotherapy and radiotherapy for advanced pancreatic cancer. The Cochrane Database of Systematic Reviews, 2018(3) https://doi.orh/10.1002/14651858.CD011044.pub $\underline{2}$

75. Shukla, S. K., Purohit, V., Mehla, K., Gunda, V., Chaika, N. V., Vernucci, E., et al. (2017). MUC1 and HIF-1alpha signaling crosstalk induces anabolic glucose metabolism to impart gemcitabine resistance to pancreatic cancer. Cancer Cell, 32(1), 71-87.e7.

https://doi.org/10.1016/j.ccell.2017.06.004
76. Krulikas, L. J., McDonald, I. M., Lee, B., Okumu, D. O., East, M. P., Gilbert, T. S. K., et al. (2018). Application of integrated drug screening/kinome analysis to identify inhibitors of gemcitabineresistant pancreatic cancer cell growth. SLAS Discovery, 23(8), 850-861. https://doi.org/10.1177/2472555218773045

77. Amrutkar, M., Aasrum, M., Verbeke, C. S., \& Gladhaug, I. P. (2019). Secretion of fibronectin by human pancreatic stellate cells promotes chemoresistance to gemcitabine in pancreatic cancer cells. BMC Cancer, 19(1), 596. https://doi.org/10.1186/s12885-019-5803-1

78. Amrutkar, M., Vethe, N. T., Verbeke, C. S., Aasrum, M., Finstadsveen, A. V., Sántha, P., et al. (2020). Differential gemcitabine sensitivity in primary human pancreatic cancer cells and paired stellate cells is driven by heterogenous drug uptake and processing. Cancers, 12(12)

https://doi.org/10.3390/cancers12123628

79. Dalin, S., Sullivan, M. R., Lau, A. N., GraumanBoss, B., Mueller, H. S., Kreidl, E., et al. (2019). Deoxycytidine release from pancreatic stellate cells promotes gemcitabine resistance. Cancer Research, 79(22), 5723-5733. https://doi.org/10.1158/00085472.CAN-19-0960

80. Hwang, H. J., Oh, M., Lee, D. W., \& Kuh, H. (2019). Multiplex quantitative analysis of stromamediated cancer cell invasion, matrix remodeling, and drug response in a 3D co-culture model of pancreatic tumor spheroids and stellate cells. Journal of Experimental E Clinical Cancer Research: CR, 38(1), 258. https://doi.org/10.1186/s13046019-1225-9

81. Ormanns, S., Haas, M., Baechmann, S., Altendorf-Hofmann, A., Remold, A., Quietzsch, D., et al. (2016). Impact of SPARC expression on outcome in patients with advanced pancreatic cancer not receiving nab-paclitaxel: A pooled analysis from prospective clinical and translational trials. British Journal of Cancer, 115(12), 15201529. https://doi.org/10.1038/bjc.2016.355

82. Xiao, Y., Zhang, H., Ma, Q., Huang, R., Lu, J., Liang, X., et al. (2019). YAP1-mediated pancreatic stellate cell activation inhibits pancreatic cancer cell proliferation. Cancer Letters, 462, 51-60.

https://doi.org/10.1016/j.canlet.2019.07.015 
83. Kettunen, K., Boström, P. J., Lamminen, T., Heinosalo, T., West, G., Saarinen, I., et al. (2019). Personalized drug sensitivity screening for bladder cancer using conditionally reprogrammed patientderived cells. European Urology, 76(4), 430-434. https://doi.org/10.1016/j.eururo.2019.06.016

84. Svirshchevskaya, E., Doronina, E., Grechikhina, M., Matushevskaya, E., Kotsareva, O., Fattakhova, G., et al. (2019). Characteristics of multicellular tumor spheroids formed by pancreatic cells expressing different adhesion molecules. Life Sciences (1973), 219, 343-352.

https://doi.org/10.1016/j.1fs.2019.01.034

85. Frappart, P., \& Hofmann, T. G. (2020). Pancreatic ductal adenocarcinoma (PDAC) organoids: The shining light at the end of the tunnel for drug response prediction and personalized medicine. Cancers, 12(10) https://doi.org/10.3390/cancers12102750

86. Lee, J., Kim, S., Khawar, I. A., Jeong, S., Chung, S., \& Kuh, H. (2018). Microfluidic co-culture of pancreatic tumor spheroids with stellate cells as a novel 3D model for investigation of stromamediated cell motility and drug resistance. Journal of Experimental \& Clinical Cancer Research : CR, 37 https://doi.org/10.1186/s13046-017-0654-6

87. Tiriac, H., Belleau, P., Engle, D. D., Plenker, D., Deschênes, A., Somerville, T. D. D., et al. (2018). Organoid profiling identifies common responders to chemotherapy in pancreatic cancer. Cancer Discovery, 8(9), 1112-1129. https://doi.org/10.1158/2159-8290.cd-18-0349

88. Wang, B., Shen, C., Li, Y., Zhang, T., Huang, H., Ren, J., et al. (2019). Oridonin overcomes the gemcitabine resistant $\mathrm{PANC}-1 /$ gem cells by regulating GST pi and LRP/1 ERK/JNK signalling. OncoTargets and Therapy, 12, 57515765. https://doi.org/10.2147/OTT.S208924

89. Wei, L., Ye, H., Li, G., Lu, Y., Zhou, Q., Zheng, S., et al. (2018). Cancer-associated fibroblasts promote progression and gemcitabine resistance via the $S D F-$ 1/SATB-1 patbway in pancreatic cancer Springer Science and Business Media LLC. https://doi.org/10.1038/s41419-018-1104-x

90. Guo, L., Zheng, J., Yu, T., Liu, Y., \& Duo, L. (2017). Elevated expression of SATB1 is involved in pancreatic tumorigenesis and is associated with poor patient survival. Molecular Medicine Reports, 16(6), 8842-8848. https://doi.org/10.3892/mmr.2017.7683

91. Chen, Z., Li, Z., Li, W., Zong, Y., Zhu, Y., Miao, Y., et al. (2015). SATB1 promotes pancreatic cancer growth and invasion depending on MYC activation Springer Science and Business Media LLC. https://doi.org/10.1007/s10620-015-3759-9

92. Dandawate, P., Kaushik, G., Ghosh, C., Standing, D., Ali Sayed, A. A., Choudhury, S., et al. (2020). Diphenylbutylpiperidine antipsychotic drugs inhibit prolactin receptor signaling to reduce growth of pancreatic ductal adenocarcinoma in mice. Gastroenterology, 158(5), 1433-1449.e27. https://doi.org/10.1053/j.gastro.2019.11.279

93. Daher, B., Parks, S. K., Durivault, J., Cormerais, Y., Baidarjad, H., Tambutte, E., et al. (2019). Genetic ablation of the cystine transporter $\mathrm{xCT}$ in PDAC cells inhibits mTORC1, growth, survival, and tumor formation via nutrient and oxidative stresses. Cancer Research, 79(15), 3877-3890. https://doi.org/10.1158/0008-5472.CAN-18-3855

94. Mattie, M., Christensen, A., Chang, M. S., Yeh, W., Said, S., Shostak, Y., et al. (2013). Molecular characterization of patient-derived human pancreatic tumor xenograft models for preclinical and translational development of cancer therapeutics. Neoplasia (New York, N.Y.), 15(10), 1138-1150. https://doi.org/10.1593/neo.13922

95. Tang, B., Yang, Y., Kang, M., Wang, Y., Wang, Y., Bi, Y., et al. (2020). m6A demethylase ALKBH5 inhibits pancreatic cancer tumorigenesis by decreasing WIF-1 RNA methylation and mediating wnt signaling. Molecular Cancer, 19(1), 3. https://doi.org10.1186/s12943-019-1128-6

96. Wei, X., Yang, J., Adair, S. J., Ozturk, H., Kuscu, C., Lee, K. Y., et al. (2020). Targeted CRISPR screening identifies PRMT5 as synthetic lethality combinatorial target with gemcitabine in pancreatic cancer cells. Proceedings of the National Academy of Sciences of the United States of America, 117(45), 28068-28079. https://doi.org/10.1073/pnas.2009899117

97. Lee, J. W., Komar, C. A., Bengsch, F., Graham, K., \& Beatty, G. L. (2016). Genetically engineered mouse models of pancreatic cancer: The KPC model (LSL-KrasG12D/+;LSL- 
Trp53R172H/+;pdx-1-cre), its variants and their application in immuno-oncology drug discovery. Current Protocols in Pharmacology / Editorial Board, S.J. Enna (Editor-in-Chief) ... [Et Al.], 73, 14.39.114.39.20. https://doi.org/10.1002/cpph.2

98. Westphalen, C. B., \& Olive, K. P. (2012). Genetically engineered mouse models of pancreatic cancer. Cancer Journal (Sudbury, Mass.), 18(6), 502510.

https://doi.org/10.1097/PPO.0b013e31827ab4c4

99. Olive, K. P., \& Tuveson, D. A. (2006). The use of targeted mouse models for preclinical testing of novel cancer therapeutics. Clinical Cancer Research, 12(18), 5277-5287.

100. Tadros, S., Shukla, S. K., King, R. J., Gunda, V., Vernucci, E., Abrego, J., et al. (2017). De novo lipid synthesis facilitates gemcitabine resistance through endoplasmic reticulum stress in pancreatic cancer. Cancer Research, 77(20), 5503-5517. https://doi.org/10.1158/0008-5472.CAN-16-3062

${ }^{101 .}$ Buchholz, S. M., Goetze, R. G., Singh, S. K., Ammer-Herrmenau, C., Richards, F. M., Jodrell, D. I., et al. (2020). Depletion of macrophages improves therapeutic response to gemcitabine in murine pancreas cancer. Cancers, 12(7) https://doi.org/10.3390/cancers12071978

102. Aiello, N. M., Bajor, D. L., Norgard, R. J., Sahmoud, A., Bhagwat, N., Pham, M. N., et al. (2016). Metastatic progression is associated with dynamic changes in the local microenvironment. Nature Communications, 7, 12819. https://doi.org/10.1038/ncomms12819

103. Principe, D. R., Narbutis, M., Kumar, S., Park, A., Viswakarma, N., Dorman, M. J., et al. (2020). Long-term gemcitabine treatment reshapes the pancreatic tumor microenvironment and sensitizes murine carcinoma to combination immunotherapy. Cancer Research, 80(15), 3101-3115. https://doi.org/10.1158/0008-5472.CAN-19-2959

104. Halbrook, C. J., Pontious, C., Kovalenko, I., Lapienyte, L., Dreyer, S., Lee, H., et al. (2019). Macrophage-released pyrimidines inhibit gemcitabine therapy in pancreatic cancer. Cell Metabolism, 29(6), 1390-1399.e6. https://doi.org/10.1016/j.cmet.2019.02.001 105. Özdemir, B. C., Pentcheva-Hoang, T., Carstens, J. L., Zheng, X., Wu, C., Simpson, T., et al.
(2014). Depletion of carcinoma-associated fibroblasts and fibrosis induces immunosuppression and accelerates pancreas cancer with diminished survival. Cancer Cell, 25(6), 719-734. https://doi.org/10.1016/j.ccr.2014.04.005

106. Zhu, X., Shen, X., Qu, J., Straubinger, R. M., \& Jusko, W. J. (2018). Proteomic analysis of combined gemcitabine and birinapant in pancreatic cancer cells. Frontiers in Pharmacology, 9, 84. https://doi.org/10.3389/fphar.2018.00084

107. Chen, Y., Liu, J., Lin, S., Li, J., Huang, S., Chen, J., et al. (2011). Proteomic analysis of gemcitabineinduced drug resistance in pancreatic cancer cells. Molecular bioSystems, 7(11), 3065-3074. https://doi.org/10.1039/c1mb05125c

108. Law, H. C. -., Lagundžin, D., Clement, E. J., Qiao, F., Wagner, Z. S., Krieger, K. L., et al. (2020). The proteomic landscape of pancreatic ductal adenocarcinoma liver metastases identifies molecular subtypes and associations with clinical response. Clinical Cancer Research: An Official Journal of the American Association for Cancer Research, 26(5), 1065-1076. https://doi.org/10.1158/1078-0432.CCR-19-1496

109. Yamamoto, K. N., Nakamura, A., Liu, L. L., Stein, S., Tramontano, A. C., Kartoun, U., et al. (2019). Computational modeling of pancreatic cancer patients receiving FOLFIRINOX and gemcitabine-based therapies identifies optimum intervention strategies. PloS One, 14(4), e0215409. https://doi.org/10.1371/journal.pone.0215409

110. Dominguez, C. X., Müller, S., Keerthivasan, S., Koeppen, H., Hung, J., Gierke, S., et al. (2020). Single-cell RNA sequencing reveals stromal evolution into LRRC15+ myofibroblasts as a determinant of patient response to cancer immunotherapy. Cancer Discovery, 10(2), 232-253. https://doi.org/10.1158/2159-8290.CD-19-0644

111. Hassel, B. A., Guyal, G., Lee, E., SontheimerPhelps, A., Levy, O., Chen, C. S., \& Ingber, D. E. (2017). Human Organ Chip Models Recapitulate Orthotopic Lung Cancer Growth, Therapeutic Responses, and Tumor Dormancy In Vitro, Cell reports, 21(2), 508-516. https://doi.org/10.1016/j.celrep.2017.09.043 



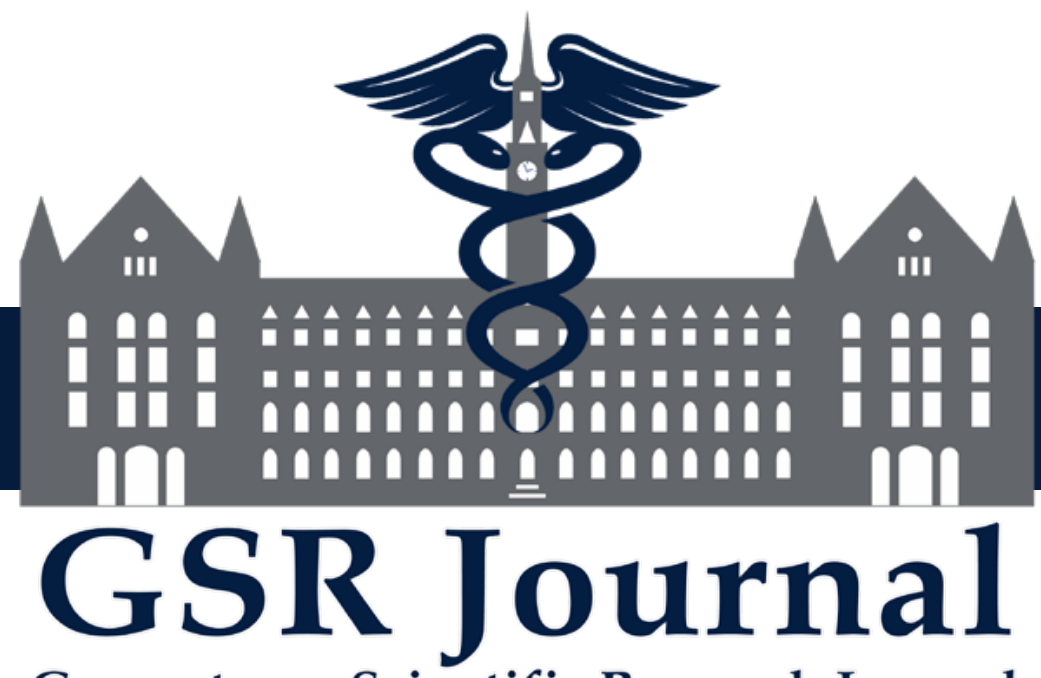

Georgetown Scientific Research Journal 